\title{
LXXVIII. On the bending of electric waves round the earth. III
}

\author{
J.W. Nicholson M.A. D.Sc.
}

To cite this article: J.W. Nicholson M.A. D.Sc. (1910) LXXVIII. On the bending of electric waves round the earth. III, Philosophical Magazine Series 6, 19:113, 757-760, DOI:

$10.1080 / 14786440508636854$

To link to this article: http://dx.doi.org/10.1080/14786440508636854

曲 Published online: 21 Apr 2009.

Submit your article to this journal \lceil

Џ Article views: 3

Q View related articles $\square$

Citing articles: 1 View citing articles $\square$ 
secondary radiation, apparently generated by lead and capable of penetrating readily all substances, except lead, even after reflexion from wood \&c.

(6) The initial variations in the absorption coefficient of the radium $\gamma$-rays have been shown to depend much on the nature of the absorber and on the disposition employed. Zine, tin, and aluminium, for a certain disposition, absorbed the rays quite exponentially but with somewhat high values of $\lambda$. In another disposition zinc absorbed exponentially with normal value of $\lambda$ from the thickness sufficient to eliminate $\beta$-rays up to $6 \mathrm{~cm}$.

(7) The value of the absorption coefficient, over the higher ranges of thickness for which absorption is always strictly exponential, can be varied within fairly wide limits, being for example always diminished when the rays first traverse a denser substance ("hardening"). The abnormal ratio $\lambda_{\mathrm{Ur}_{\mathrm{r}}} / \lambda_{\mathrm{Ra}}$ for lead $(1 \cdot 46.5)$ previously obtained is so accounted for. In general, the ratio $\lambda_{\mathrm{Ur}} \mathrm{x} / \lambda_{\mathrm{Ra}}$ may be taken to be from $1 \cdot 2$ to $1 \cdot 3$.

Physical Chemistry Laboratory, Glasgow University.

LXXVIII. On the Bending of Electric Waves round the Earth. III. By J. W. Nicholson, M.A., D.Sc.*

T $N$ the second note on this subject $\dagger, M$. Poincaré's second investigation was considered, and it was indicated that when a radial oseillator is placed close to the earth, regarded as a perfect conductor, the effect produced by diffraction at any point in the geometrical shadow can only differ from an exponential type with large negative argument by a series of inverse powers of $k a$, where $k a$ is a magnitude roughly of order $10^{-6}$. In the meantime, the writer has continued the examination of these "residual" terms depending on harmonics of low order, and bas proved that they do in fact have a zero sum for points on the surface of the sphere. They play their part in the remarkable compensation of the terms of the harmonic series, so that the total effect at a point on the surface is, in fact, exponential. M. Poincare's exponential factor is thus valid for surface points, although his mode of investigation in its present form does not apply to points not on the surface, even though fairly close to it. The proof of the evanescence of these terms in inverse

* Communicated by the Anthor.

† Phil. Mag. March 1910. 
power of $k a$ will appear in the writer's second paper "On the bending of electric waves round a large sphere."

But very different results have been obtained by Prof. $H$. M. Macdonald in a paper just published ${ }^{*}$, and the main purpose of this note is an examination of Prof. Macdonald's proof, in which there appears to be a flaw which gives rise to the whole difference in the results. The problem is treated first in its general case, in which the oscillator is not close to the surface, and the effect at any point whatever is investigated to a first approximation. In an appendix, the main formulæ of pure mathematics required for the solution are given. These relate, in the first place, to the summation of a type of oscillating series, and in this section the summation follows the lines of that given bv the writer previously $\dagger$, and particular cases of the general formula are developed.

The remainder of the appendix is devoted to an examination of asymptotic values of the Bessel functions, in which results are obtained in accord with those of the papers mentioned in previous notes.

In the treatment of the actual physical problem, Prof. Macdonald first shows that the principal value of the sum of the harmonic series at all points not very close to the geometrical shadow and outside it, is identical with that which would be obtained by the methods of geometrical optics, provided that certain terms are neglected which, as is stated in a footnote, are only important in the neighbourhood of the shadow. But when he proceeds to an examination of the shadow, the method employed is liable to a criticism which appears to be very decisive. In accordance with the usage of the writer's first paper on the subject $f$, a point at which the derivate of an exponent in an oscillating harmonic series can vanish will be called a "zero point." Prof. Macdonald reduces the problem in this case to a determination of the principal part of a series of the form, where $A$ is the same for every term,

$$
\mathrm{S}_{43}=\mathrm{A} \Sigma_{z_{0}}^{\infty} m^{\frac{3}{2}}\left(\mathrm{RR}_{1}\right)^{\frac{1}{2}} e^{-1\left(\phi+\phi_{1}+m \theta-\frac{1}{4} \pi\right)} \quad . \quad .
$$

$m$ being $n+\frac{1}{2}$. The functions $\mathrm{R}$ and $\mathrm{R}_{1}$ do not oscillate in any part of the range of summation. The orientation of the point considered is $\theta$, and the distances of this point and

* Phil. Trans. A. vol. cex. pp. 113-144.

+ Messenger of Math., Oct. 1907.

$\ddagger$ Phil. Mag. April 1910. 
of the oscillator from the centre of the earth of radins $a$ are respectively $r$ and $r_{1}$. If $2 \pi / k$ be the wave-length, $z=k r$, $z_{0}=k a, z_{1}=k r_{1}$, and $\phi$ and $\phi_{1}$ are similar functions of $z$ and $z_{1}$ involving $m$ or $n+\frac{1}{2}$. The forms taken by these functions are determined by the relative values of $m$ and their arguments. Prof. Macdonald then writes this series as the difference of two otbers, whose summations range from 1 to $\infty$ and from 1 to $z_{0}$ respectively. These series have the same zero point, defined by

$$
\theta+\partial \phi / \partial n+\partial \phi_{1} / \partial n=0
$$

and their principal parts arise from the terms on both sides of, but close to the term for which this is true.

For simplicity, we may restrict attention to the case in which $\phi$ and $\phi_{1}$ are identical, so that the transmitter and receiver are equidistant from the centre of the earth, for this is the case in which a tabulation of the final formula is given. Now if $n$ and $\approx$ are not nearly equal,

$$
\partial \phi / \partial n=\alpha-\frac{1}{2} \pi
$$

where $\sin \alpha=\left(n+\frac{1}{2}\right) / z$, and the zero point for the case $\phi=\phi_{1}$ is given by $\theta=\pi-2 \alpha$, as in Prof. Macdonald's paper.

Now let $n_{1}$ denote the value of $n$ at this zero point. By the use of this value, which in the special case $\phi=\phi_{1}$ is given by $n_{1}+\frac{1}{2}=z \cos \frac{1}{2} \theta$, Prof. Macdonald obtains the principal parts of the two component series, so that by subtraction, $\mathrm{S}_{43}$ is proportional to the integral

$$
\left(\int_{-\infty}^{z_{0}-n_{1}-\frac{1}{2}}-\int_{-\infty}^{\infty}\right) d \zeta e^{-\frac{1}{2}, \zeta^{2} / z \cos \pi} . .
$$

where the argument of the exponential in the integrand is derived from

$$
\partial^{2} \phi / \partial n^{2}=\sec \alpha
$$

which is true when $n_{1}$ and $z$ are not too nearly equal, if also $\theta$ is not too small. But this condition has already been introduced with the use of an asymptotic formula for a zonal harmonic. This integral is finally reduced to a dependence on Fresnel's integrals in the form

where

$$
\int_{\eta_{0}}^{\infty} d \eta e^{-\frac{1}{2} \pi \iota \eta^{2}} \quad \cdot \quad \cdot \quad \cdot \quad \cdot
$$

$$
\eta_{0}=2\left(a-r \cos \frac{1}{2} \theta\right)\left(\lambda r \sin \frac{1}{2} \theta\right)^{-\frac{1}{2}} . . .
$$

for the case of an oscillator close to the earth, $\lambda$ being the wave-length. 
Now the objection to be urged is that this formula cannot be used for points near the earth's surface. For other points not very close, it does lead to the true sum for the series $S_{43}$, and, as Prof. Macdonald remarks, is in agreement with a previous result. But when the oscillator and receiver are both close to the surface, $z$ and $z_{1}$ are both practically equal to $z_{0}$, and consequently $\phi$ and $\phi_{1}$ equal to $\phi_{0}$ for any value of $n$. The original series (2) commences with the harmonic of order $z_{0}$ for which $\phi_{0}$, now the common value of the functions $\phi$, is not of high order and in fact is practically equal to $\frac{1}{6} \pi$. At no point in the series is $\phi$ of a form in accordance with (3). Now Prof. Macdonald has replaced this series by two others each commencing with the harmonic of order unity. In each of these, $\phi$ can take a form consistent with (3) for part of the range, and they both lead to the same zero point. But it is not justifiable to take, as the sum of the original series, the difference in the principal values of these two series. For in determining a principal value, it is definitely supposed that there are a large number of harmonics on each side of the zero point for which $\phi, \phi_{1}$ (and, moreover, $\mathrm{R}$ and $\mathrm{R}_{1}$ and similar functions) do not change in type, and the changes of type, for harmonics beyond these, are ignored. But in the present case, it is harmonics of different type which alone are concerned, as we have seen, since the original sum extended from $z_{0}$ to $\infty$. It cannot therefore be lawful to equate their sum to the difference of two series (from 1 to $z_{0}$, and from 1 through $z_{0}$ to $\infty$ ) deduced by a method which assumes, even in the second of these series, that the harmonics continue to $\infty$ of the same type, a type not found in the original series.

These considerations appear to show definitely that the formula given by Prof. Macdonald is not valid when the transmitter and receiver are near the earth. Moreover, it will be seen that when $\phi$ and $\phi_{1}$ are not nearly equal to $\phi_{0}$, a large number of harmonics of the proper type are found in the original series ( 1 ), and the formula thus becomes valid. 'This explains why, for points not close to the shadow, it is in accord with previous results.

We must conclude finally that the tables given for the intensity produced by diffraction round the surface onght to exhibit a very mueh smaller effect. In a later section of the paper now being published " On the bending of electric waves round a large sphere," tables of the exponential formula will be given. In the form in which M. Poincare has left it, there are two undetermined magnitudes. 